\title{
A comparative study on superb microvascular imaging and conventional ultrasonography in differentiating BI-RADS 4 breast lesions
}

\author{
YI-CHENG ZHU ${ }^{1}$, DAO-MING ZU ${ }^{2}$, YUAN ZHANG ${ }^{1}$, JUN SHAN ${ }^{1}$, \\ XIU-RONG SHI ${ }^{1}$, SHU-HAO DENG ${ }^{1}$ and QUAN JIANG ${ }^{1}$ \\ Departments of ${ }^{1}$ Ultrasound and ${ }^{2}$ Minor Surgery, Pudong New Area People's Hospital Affiliated to \\ Shanghai University of Medicine and Health Sciences, Shanghai 201299, P.R. China
}

Received October 20, 2018; Accepted June 11, 2019

DOI: $10.3892 / \mathrm{ol} .2019 .10603$

\begin{abstract}
This prospective study aimed to explore the diagnostic value of superb microvascular imaging (SMI) in differentiating Breast Imaging Reporting and Data System (BI-RADS) 4 breast lesions compared with conventional ultrasonography (US). A total of 111 patients with 116 breast lesions underwent grayscale ultrasound (US), colour Doppler flow imaging (CDFI) and SMI breast imaging between February 2016 and May 2018. CDFI and SMI were performed to evaluate vascular quantity, morphology, and distribution characteristics. The detection of malignancy was compared between grayscale US alone, US + CDFI and US + SMI in terms of the BI-RADS stratification system. SMI was observed to be significantly more accurate in distinguishing malignant breast lesions (86.67\%) compared with CDFI $(80.00 \%)$ $(\mathrm{P}<0.001)$. Among malignant lesions, SMI detected $80.00 \%$ of those that contained $\geq 4$ vessels, while CDFI only detected $56.67 \%$. Penetrating and branching vessels were identified by SMI in $53.33 \%$ of malignant breast lesions and by CDFI in $10.00 \%$. There was no significant difference in vascular distribution by SMI $(\mathrm{P}=0.094)$ and by CDFI $(\mathrm{P}=0.087)$. US + SMI was associated with higher sensitivity, specificity, and accuracy rates $(86.67,83.72$ and $84.48 \%$, respectively) compared
\end{abstract}

Correspondence to: Dr Quan Jiang, Department of Ultrasound, Pudong New Area People's Hospital Affiliated to Shanghai University of Medicine and Health Sciences, 490 South Chuahuan Road, Shanghai 201299, P.R. China

E-mail: quan_JMD@126.com

Abbreviations: US, ultrasonography; CDFI, color Doppler flow imaging; SMI, superb microvascular imaging; BI-RADS, Breast Imaging Reporting and Data System; US-FNA, ultrasound-guided fine needle aspiration; ROC, receiver-operating characteristic; AUC, area under the curve

Key words: superb microvascular imaging, breast lesions, colour Doppler flow imaging, ultrasound, breast imaging reporting, data system with US + CDFI (80.00, 72.09 and $74.14 \%$, respectively). The area under the curve values from receiver operating characteristic analysis of US + SMI, US + CDFI and US alone were 0.852 [95\% confidence interval (CI): 0.768-0.936)] 0.760 (95\% CI: 0.660-0.860), 0.698 (95\% CI: 0.589-0.807), respectively $(\mathrm{P}<0.001)$. SMI yielded more detailed vascular information associated with malignant breast masses when compared with conventional US. Therefore, as an adjunct to grayscale, SMI exhibited a markedly improved diagnostic capability in distinguishing malignant and benign breast lesions, particularly those of BI-RADS category 4.

\section{Introduction}

Breast cancer has been among the leading causes of cancer-associated mortality among Chinese women in the past ten years (1). The 5-year survival rates vary from $100 \%$ (Stage I) to $22 \%$ (Stage IV) (2); thus, early diagnosis is crucial. Tumor growth has been demonstrated to be largely dependent on angiogenesis (3). The formation of new blood vessels serves a pivotal role in the local growth, invasion and distant metastasis of breast cancer (4). Therefore, this identifying this distinct characteristic between malignant and benign breast lesions may represent a supplementary method for improving diagnostic performance and accuracy. Color Doppler flow imaging (CDFI) and power Doppler flow imaging have been extensively applied in clinical practice $(5,6)$. However, such non-invasive methods adjunct to grayscale ultrasonography (US) exhibit low sensitivity in detecting microvascularity (7), and are largely dependent on the mean Doppler frequency shift, with inevitable loss of low-velocity blood flow information. By contrast, superb microvascular imaging (SMI), an emerging Doppler US method, suppresses clutter to delineate a wider range of blood flow signals with a higher resolution. Therefore, SMI is capable of detecting low-velocity and high-velocity flow, while CDFI is unable to detect very low-flow states due to the different blood flow extraction principles (8). Previous trials have confirmed the higher sensitivity of SMI in depicting central and peripheral vessels in hepatic lesions, compared with CDFI $(9,10)$.

The American College of Radiology first introduced the Breast Imaging Reporting and Data System (BI-RADS) 
in 2003, which was updated in 2013 (11). This reporting system includes a wide range of US findings for malignancy classifications, such as shape and echogenicity pattern and posterior acoustic characteristics. The updated version added subdivisions to category 4 , taking into consideration the fact that BI-RADS category 4 is the most controversial (12). The likelihood of malignancy in that stratification ranges from 2-95\% (13), as BI-RADS category 4 breast masses are affected by a diverse range of clinical factors such as abscess, hematoma and fat necrosis (14).

To the best of our knowledge, few studies have applied the SMI technique to differentiate the malignancy among BI-RADS 4 breast lesions. Therefore, the aim of the present study was to explore the diagnostic value of SMI in differentiating between malignant and benign BI-RADS 4 breast lesions compared with conventional US.

\section{Patients and methods}

Clinical data. Between February 2016 and May 2018, 291 patients were diagnosed with breast lesions detected by conventional US in our hospital. In total, 116 lesions in 111 patients (age range, 16-64 years; mean age, $47.13 \pm 9.29$ years) were recruited in this prospective study. Among the 111 patients, 5 presented with 2 lesions. The flowchart of the selection process is presented in Fig. 1 and the inclusion criteria were as follows: i) Breast lesion detectable by US; ii) breast lesion was classified as BI-RADS 4 category; and iii) no history of treatment prior to US examination. The exclusion criteria were as follows: i) Incomplete data $(n=49)$; and ii) breast surgery or chemotherapy for the same lesions $(n=9)$. The mean diameter of the lesions was $17.93 \pm 8.51 \mathrm{~mm}$ (range, 8.0-48.3 mm), while the mean depth was $26.45 \pm 11.35 \mathrm{~mm}$ (range, 8.1-47.3 mm). All lesions were pathologically confirmed by a US-guided core needle biopsy and/or surgery according to standard clinical protocols. This prospective study was approved by the Ethics Committee of Shanghai Pudong New Area People's Hospital (Shanghai, China). All patients in the present study were provided with information on all the examinations and procedures and provided written informed consent to participate in the study.

Equipment and methods. All patients initially underwent a grayscale US examination, using a TOSHIBA Aplio 500 (Toshiba Medical System Corporation, Tokyo, Japan) with high-frequency $(14 \mathrm{MHz})$ line array transducers. When a breast lesion was detected, the lesion size, depth, shape and other US characteristics, including the margin, echogenicity and posterior acoustic elements were recorded. Grayscale US was followed by CDFI (frame rate 10-15 Hz) and SMI (frame rate $>50 \mathrm{~Hz}$ ) to evaluate vascular quantity, morphology and distribution. The velocity scope of SMI was adapted to $<2.5 \mathrm{~cm}$ per second. Gentle pressure was applied through the transducer to prevent vessel collapsing. During the examination, patients were placed in the supine position with the arms elevated and were instructed to breathe calmly.

The same radiologist ( $Y C Z$ ) with $>3$ years of experience in breast US and 1-year experience in SMI conducted all examinations. The same imaging area for CDFI and SMI vascular blood flow images were acquired as the reference area for the breast tissue (Figs. 2 and 3). All images were recorded and transferred to the hospital's internal online database. The images were evaluated by two radiologists (YZ and SHD), who had 10- and 5-years respective experience in CDFI and breast imaging, and 2 years in SMI. A two-stage rating process was applied to the imaging of the breast lesions. Each breast lesion was first rated according to BI-RADS based on the aforementioned US characteristics; thereafter, vascular quantity, morphology and distribution were rated based on CDFI and SMI findings. Vascular quantity was graded according to the Adler's classification (15) as follows: i) Absent, grade 0; ii) minimal, grade 1 ; iii) moderate, grade 2 ; or iv) marked, grade 3 , dependent on the amount of blood flow in the region of interest (15). Grade 0 referred to no blood flow detected; minimal (grade 1) flow generally referred to 1 or 2 pixels containing flow $(<0.1 \mathrm{~cm}$ in diameter); moderate (grade 2 ) referred to a certain number of small vessels and/or a main vessel and marked (grade 3 ) vascularity was defined as $\geq 4$ vessels visualized (15). Morphological characteristics were evaluated using a classification that included 7 categories, including not applicable (N/A), linear, dot-like, penetrating, branching, penetrating and branching, and shunt. Vessel distribution was further divided into three categories, peripheral, central, and both peripheral and central, respectively. Based on these findings, the two aforementioned radiologists then re-corrected BI-RADS stratification. If any disagreement occurred, a third senior radiologist (QJ), with an experience of $>15$ years in breast US and 2 years in SMI, was consulted. All radiologists were blinded to the pathological findings.

Statistical analysis. The $\chi^{2}$ test or Fisher's exact tests were applied for categorical variables, while an independent-samples t-test was applied for the comparison of continuous variables. Quantitative data are expressed as mean \pm standard deviation when normally distributed. The findings on new vessel formation from CDFI and SMI were compared between the malignant and benign lesions using $\chi^{2}$ test. A receiver operating characteristics (ROC) curve was formulated to determine the diagnostic value of CDFI and SMI. The areas under the curves (AUCs) of different diagnostic modalities were compared using the $\chi^{2}$ test. Statistical analysis was performed using the pathological results as the diagnostic gold standard. $\mathrm{P}<0.05$ was considered to indicate statistically significant differences. Data analysis was performed using SPSS 25.0 software (IBM Corp., Armonk, NY, USA).

\section{Results}

US characteristics. Of the 116 breast lesions examined, 30 $(25.9 \%)$ lesions were pathologically confirmed as malignant. The malignant lesions were further categorized as invasive ductal carcinomas $(\mathrm{n}=17)$, ductal carcinoma in situ $(\mathrm{n}=6)$, tubular carcinomas $(n=2)$, invasive lobular carcinomas $(n=2)$ and mucinous carcinomas $(n=3)$. Among the 86 benign lesions, the most common benign pathological result was fibrocystic change $(n=24)$, followed by fibroadenoma $(n=23)$ and ductal hyperplasia $(n=18)$. The pathological results of the breast masses are summarized in Table I. Conventional US characteristics, such as irregular shape and non-circumscribed margins [malignant, 93.3\% (28/30); benign, 77.9\% (67/86)], 


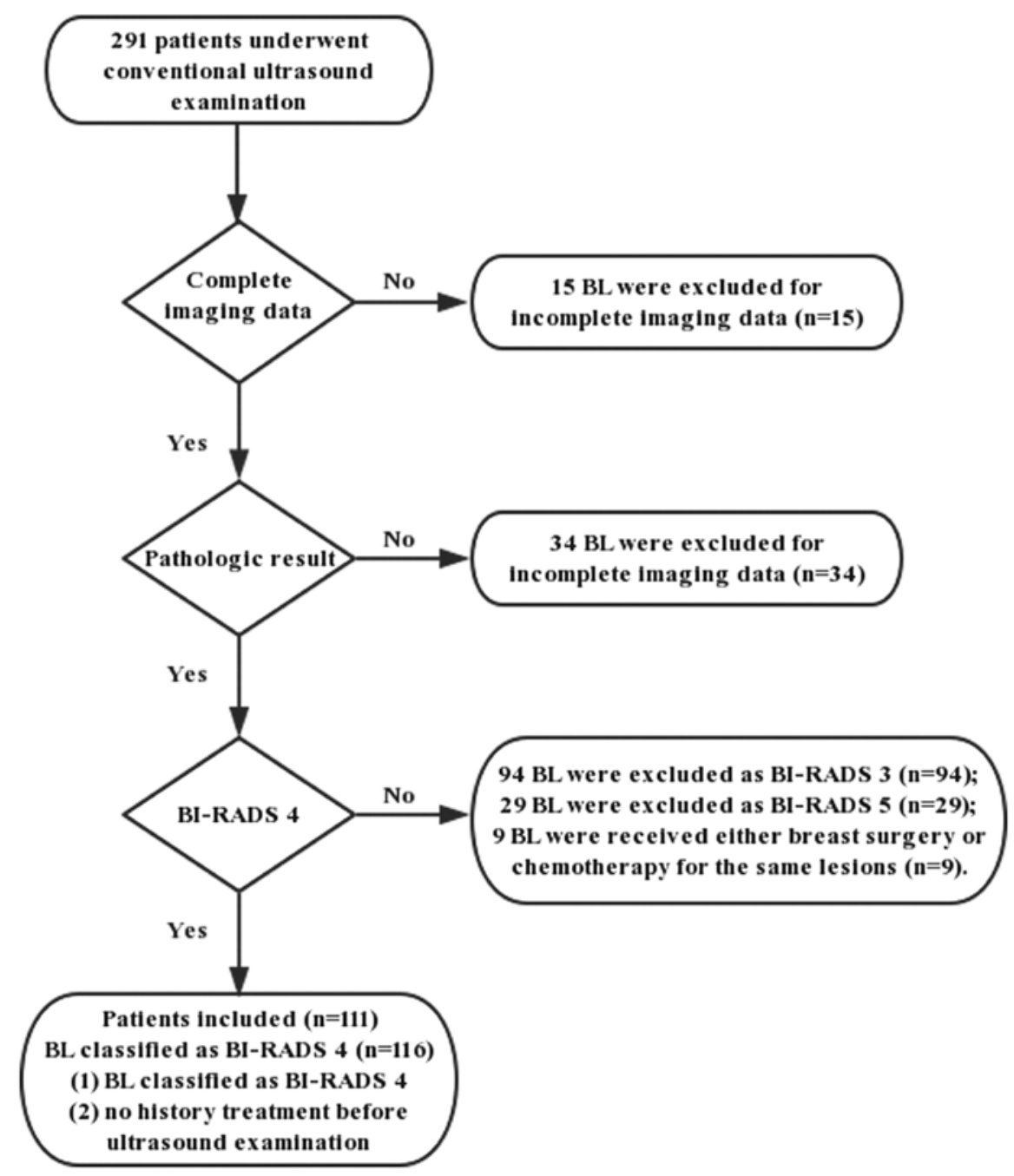

Figure 1. Flowchart of patient selection. BI-RADS, Breast Imaging Reporting and Data System; BL, benign lesion.

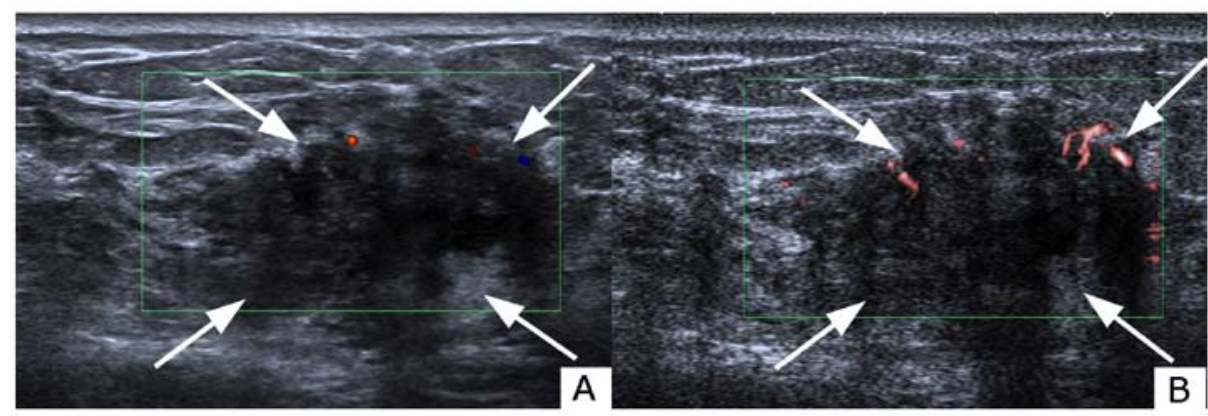

Figure 2. Evaluation of blood build-up in a lesion of the right breast with (A) CDFI and (B) SMI in a 61-year-old female. Grading was grade 1 with CDFI and grade 3 with SMI. Analysis of the morphology revealed a penetrating and branching pattern under SMI examination. The vessel distribution was peripheral and central. The lesion was classified as ultrasonography BI-RADS category 4a under CDFI and category $4 \mathrm{c}$ under SMI. The lesion was pathologically determined as invasive ductal carcinoma. The white arrows indicate the lesion shown on CDFI and SMI. The red and blue areas represent vascularity. The difference between red and blue signals shown on CDFI denotes blood flow distribution. Red areas depict blood flow towards the probe, whereas the blue areas depict blood flow away from the probe. CDFI, color Doppler flow imaging; SMI, superb microvascular imaging.

were more commonly identified in malignant breast masses when compared with benign breast lesions $(\mathrm{P}<0.05$; Table II).

Evaluation of microvasculature parameters in breast lesions using CDFI and SMI. The vascular quantity was evaluated using Adler's classification, as shown in Table III. CDFI and
SMI exhibited a noticeable variance between malignant and benign masses $(\mathrm{P}<0.001)$. CDFI identified 21 breast masses as avascular, while SMI revealed absent vascularity in 17 of those masses, which indicated that SMI was more efficient in distinguishing microvessels. Based on the identification of the microvasculature, SMI detected $80.00 \%$ of malignant lesions 
Table I. Pathological results of all BI-RADS 4 category breast lesions, [n (\%)].

\begin{tabular}{lc}
\hline Lesions & $\mathrm{n}(\%)$ \\
\hline Malignant & $30(25.9)$ \\
Invasive ductal carcinoma & $17(56.7)$ \\
Ductal carcinoma in situ & $6(20.0)$ \\
Tubular carcinoma & $2(6.7)$ \\
Invasive lobular carcinoma & $2(6.7)$ \\
Mucinous carcinoma & $3(10.0)$ \\
Benign & $86(74.1)$ \\
Papilloma & $7(8.1)$ \\
Fibroadenoma & $23(26.7)$ \\
Fibrocystic change & $24(27.9)$ \\
Ductal hyperplasia & $18(20.9)$ \\
Sclerosing adenosis & $3(3.5)$ \\
Columnar cell lesions & $11(12.8)$ \\
\hline
\end{tabular}

that contained $\geq 4$ vessels, while CDFI only identified $56.67 \%$ of malignant breast lesions with rich blood flow signals. In comparison, the majority of benign lesions were avascular (CDFI: 22.1\%; SMI: $18.6 \%$ ) or hypo-vascular (CDFI: 53.5\%; SMI: 52.3\%; Table III). Avascular lesions were rarely identified in malignant breast lesions; under CDFI examination, 2 out of 30 malignant lesions exhibited this feature, whereas SMI detected one malignant lesion with no vascularity. In addition, the morphology of the vessels differed significantly different between CDFI and SMI $(\mathrm{P}<0.01)$. Penetrating and branching vessels were detected in malignant breast lesions using CDFI and SMI (53.33 and $10.00 \%$, respectively). However, SMI exhibited higher sensitivity in detecting complex morphological characteristics in benign breast masses, while CDFI was unable to display penetrating, branching and shunt vessels in benign breast masses. In terms of vessel distribution, malignant breast lesions more frequently exhibited peripheral and both peripheral and central distribution compared with central distribution alone, whereas benign masses more frequently exhibited central and both peripheral and central distribution. However, there was no significant difference between benign and malignant breast lesions in terms of vessel distribution using either CDFI $(\mathrm{P}=0.269)$ or SMI $(\mathrm{P}=0.220)$ (Table III).

Risk of malignancy grading and diagnostic performance of $U S, U S+C D F I$ and $U S+S M I$. In terms of subdivision of the BI-RADS category 4 of breast lesions, none were downgraded by either CDFI or SMI. A total of 5 breast neoplasms were upgraded to category 5 with CDFI, while 12 breast neoplasms were upgraded to category 5 following SMI examination (Fig. 4). Regarding the 86 benign lesions, 72, 62 and 57 were graded as BI-RADS 4a under US + SMI, US + CDFI, and US examination alone. None of the benign lesions were graded as BI-RADS 5 under any of the three examinations. When breast lesions rated as BI-RADS 4a were considered as benign, and the remaining breast lesions rated as BI-RADS $4 \mathrm{~b}, 4 \mathrm{c}$ and 5 as malignant, the sensitivity, specificity and accuracy rate for SMI were $86.67,83.72$ and $84.48 \%$, respectively; the sensitivity, specificity and accuracy rate for CDFI were $80.00,72.09$ and $74.14 \%$ (Table IV). The AUC values for US, US + CDFI and US + SMI were 0.698 [95\% confidence interval (CI): $0.589-0.807], 0.760$ (95\% CI: 0.660-0.860) and 0.852 (95\% CI: 0.768-0.936), respectively (Fig. 5). There was a significant difference in the AUC value between US + CDFI and US + SMI $(\mathrm{P}<0.001)$.

\section{Discussion}

US has been widely applied as a first-line diagnostic technique in differentiating malignant and benign tumors based on the evaluation of angiogenesis and the growth of irregular vascular structures $(16,17)$. Compared with magnetic resonance imaging and contrast-enhanced US, Doppler US has the advantages of being simpler, more cost-effective and more risk-free. CDFI, one of the most widely used US techniques, provides valuable data for evaluating blood flow, but with limitation in detecting vessels $<0.1 \mathrm{~mm}$, as CDFI is generally associated with data loss due to movement artifacts attributed to the single-dimension filter (18). Due to the advances in US techniques, SMI is a pioneering technique that has the ability to visualize lower-speed bloodstream without motion artifacts (7). This advantage has been widely reported in the analysis of microvascular flow within thyroid nodules (19), testicular (20) and hepatic tumors (21). Multiple studies support the efficacy of SMI in specifically visualizing minute vessels and slow speed blood flow, with high resolution and fewer motion artifacts. After observing 123 breast masses, Ma et al (22) concluded that SMI (83.7\%) achieved a better visualization of vascularity compared with CDFI (74.8\%). The study of Zhu et al (23) evaluated microvascular blood flow in 123 breast lesions in 121 patients and demonstrated that the improved visualization of the microvasculature, including low-flow vessels, with SMI $(87.80 \%)$ when compared with CDFI $(78.05 \%)$. These studies support the findings of the present study, which demonstrated that SMI detected the presence of vessels in 99 breast masses (85.3\%), while CDFI only detected blood flow in 95 masses (81.90\%). Among avascular breast masses, only one was pathologically proven to be malignant. In the present study, malignant breast lesions tended to have $\geq 4$ vessels based on the Adler's classification. Specifically, $80.00 \%$ of the malignant breast lesions were rated as grade 3 using SMI, whereas CDFI classified only $56.67 \%$ of such lesions as grade 3 . Therefore, malignant breast neoplasms displayed more enriched flow signals. This finding was consistent with the nature of neoplastic angiogenesis, as malignant and benign lesions exhibit distinct degrees of neo-vasculature development (24). Vascularity growth is a closely associated with neoplastic proliferation (25).

In addition to vessel quantity, the present study also observed vascular morphology and distribution as potential indicators of malignancy. A high correlation between breast cancer angiogenesis, vascular morphology and distribution has been reported (26). One of the critical characteristics of malignant lesions is the presence of penetrating and complex branching patterns (27). Xiao et al (28) indicated that penetrating, spiculated or radially arranged vessels are more likely to be identified in malignant breast lesions. Zhan et al (29), 
Table II. Conventional ultrasound characteristics of benign and malignant breast lesions, [n (\%)].

\begin{tabular}{|c|c|c|c|c|}
\hline Characteristic & Malignant $(n=30)$ & Benign $(n=86)$ & Overall & P-value \\
\hline Mean age (year) & $52.43 \pm 8.12$ & $45.16 \pm 8.96$ & $47.13 \pm 9.29$ & $0.014^{\mathrm{a}}$ \\
\hline Size (diameter, mm) & $28.24 \pm 9.54$ & $14.34 \pm 4.05$ & $17.93 \pm 8.51$ & $0.014^{\mathrm{a}}$ \\
\hline Depth (mm) & $30.11 \pm 13.06$ & $25.18 \pm 10.47$ & $26.45 \pm 11.35$ & $<0.001^{\mathrm{a}}$ \\
\hline Position & & & & 0.924 \\
\hline Left & $14(46.7)$ & $41(47.7)$ & $55(47.4)$ & \\
\hline Right & $16(53.3)$ & $45(52.3)$ & $61(52.6)$ & \\
\hline Shape & & & & $<0.001^{\mathrm{a}}$ \\
\hline Oval & $5(16.7)$ & $60(69.8)$ & $65(56.0)$ & \\
\hline Round & $4(13.3)$ & $14(16.3)$ & $18(15.5)$ & \\
\hline Irregular & $21(70.0)$ & $12(14.0)$ & $33(28.4)$ & \\
\hline Margin & & & & $0.001^{\mathrm{a}}$ \\
\hline Circumscribed & $2(6.7)$ & $19(22.1)$ & $21(18.1)$ & \\
\hline Indistinct & $2(6.7)$ & $26(30.2)$ & $28(24.1)$ & \\
\hline Angular & $2(6.7)$ & $5(5.8)$ & $7(6.0)$ & \\
\hline Microlobulated & $20(66.7)$ & $30(34.9)$ & $50(43.1)$ & \\
\hline Spiculated & $4(13.3)$ & $6(7.0)$ & $10(8.6)$ & \\
\hline Orientation & & & & $0.032^{\mathrm{a}}$ \\
\hline Parallel & $16(53.3)$ & $64(74.4)$ & $80(69.0)$ & \\
\hline Non-parallel & $14(46.7)$ & $22(25.6)$ & $36(31.0)$ & \\
\hline Posterior acoustic features & & & & $<0.001^{\mathrm{a}}$ \\
\hline None & $9(30.0)$ & $57(66.3)$ & $66(56.9)$ & \\
\hline Enhancement & $3(10.0)$ & $6(7.0)$ & $9(7.8)$ & \\
\hline Shadowing & $7(23.3)$ & $23(26.7)$ & $30(25.9)$ & \\
\hline Combined pattern & $11(36.7)$ & $0(0.0)$ & $11(9.5)$ & \\
\hline Echo pattern & & & & 0.060 \\
\hline Hypoechoic & $15(50.0)$ & $54(62.8)$ & $69(59.5)$ & \\
\hline Isoechoic & $4(13.3)$ & $16(18.6)$ & $20(17.2)$ & \\
\hline Hyperechoic & $6(20.0)$ & $1(1.2)$ & $7(6.0)$ & \\
\hline Complex & $5(16.7)$ & $15(17.4)$ & $20(17.2)$ & \\
\hline
\end{tabular}

\section{${ }^{\mathrm{a}} \mathrm{P}<0.05$.}

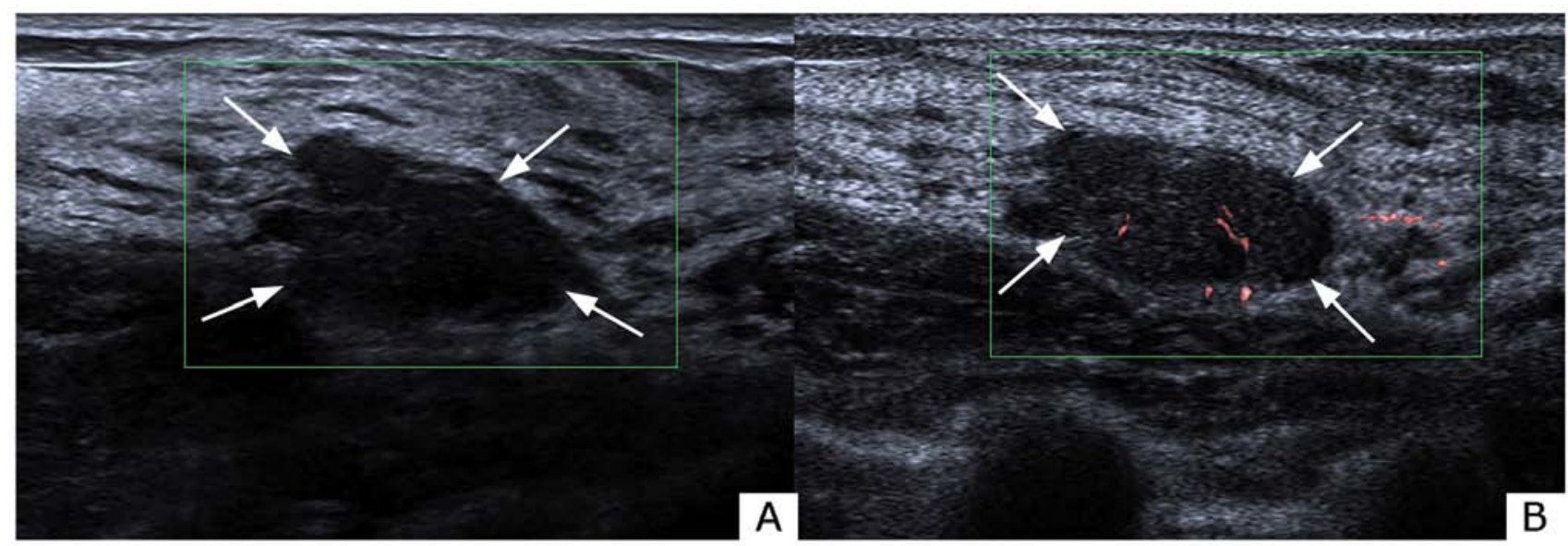

Figure 3. Evaluation of blood build-up in a lesion of the right breast with (A) CDFI and (B) SMI in a 34-year-old female. Grading was grade 0 with CDFI and grade 1 with SMI. Analysis of the morphology revealed a linear pattern under SMI examination. The vessel distribution was central. The lesion was classified as ultrasonography BI-RADS category 4a under both CDFI and SMI. The lesion was pathologically determined as fibroadenoma. The white arrows indicate the lesion shown on CDFI and SMI. The red and blue areas represent vascularity. The difference between red and blue signals shown on CDFI denotes blood flow distribution. Red areas depict blood flow towards the probe, whereas the blue areas depict blood flow away from the probe. CDFI, color Doppler flow imaging; SMI, superb microvascular imaging; BI-RADS, Breast Imaging Reporting and Data System. 

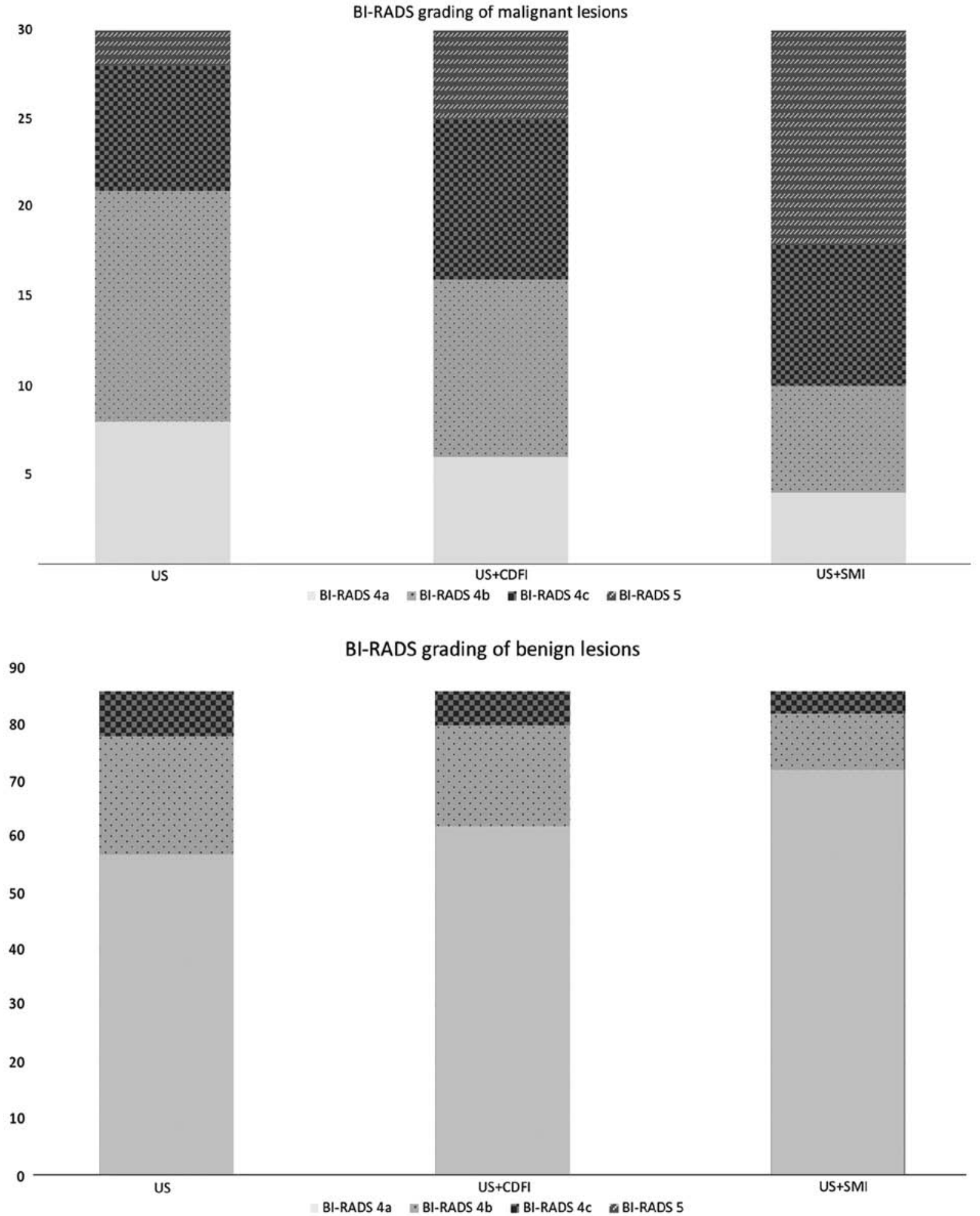

Figure 4. Correction of BI-RADS category by different technologies. BI-RADS, Breast Imaging Reporting and Data System; US, ultrasonography.

demonstrated that SMI depicted an increase in the median number of penetrating vessels when compared with CDFI. Similarly, in the present study, no shunt vessels were detected by CDFI, whereas 7 malignant breast lesions with shunt vessels were identified by SMI. With regards to the distribution of vascularity, there was no statistically significant difference between CDFI and SMI. Therefore, our findings re-confirmed that both the number and morphological characteristics of vessels are key to differentiating breast malignancies (30).
The overgrowth of immature capillaries from the vessels surrounding the lesions may explain the findings of the present study.

In the present study, the risk of malignancy was based on the BI-RADS system. SMI was demonstrated to be superior in terms of sensitivity $(86.67 \%, 26 / 30)$, specificity $(83.72 \%, 72 / 86)$ and accuracy rate $(84.48 \%, 98 / 116)$. However, the increased efficacy of SMI in extracting microvascular information may lead to an increase in false positive diagnoses. For example, 1 fibroadenoma 
Table III. Vascularity findings of malignant and benign breast lesions using CDFI and SMI, [n (\%)].

\begin{tabular}{|c|c|c|c|c|c|c|}
\hline \multirow[b]{2}{*}{ Variable } & \multicolumn{3}{|c|}{ CDFI } & \multicolumn{3}{|c|}{ SMI } \\
\hline & Malignant $(\mathrm{n}=30)$ & Benign $(n=86)$ & P-value & Malignant $(n=30)$ & Benign $(n=86)$ & P-value \\
\hline Adler classification & & & 0.009 & & & $<0.001^{\mathrm{a}}$ \\
\hline Grade 0 & $2(6.7)$ & $19(22.1)$ & & $1(3.3)$ & $16(18.6)$ & \\
\hline Grade 1 & $5(16.7)$ & $25(29.1)$ & & $2(6.7)$ & $14(16.3)$ & \\
\hline Grade 2 & $6(20.0)$ & $21(24.4)$ & & $3(10.0)$ & $31(36.0)$ & \\
\hline Grade 3 & $17(56.7)$ & $21(24.4)$ & & $24(80.0)$ & $25(29.1)$ & \\
\hline Morphology & & & $<0.001$ & & & $<0.001^{\mathrm{a}}$ \\
\hline N/A & $2(6.7)$ & $19(22.1)$ & & $1(3.3)$ & $16(18.6)$ & \\
\hline Linear & $14(46.7)$ & $44(51.2)$ & & $1(3.3)$ & $49(57.0)$ & \\
\hline Dot-like & $7(23.3)$ & $23(26.7)$ & & $2(6.7)$ & $17(19.8)$ & \\
\hline Penetrating & $2(6.7)$ & $0(0.0)$ & & $2(6.7)$ & $2(2.3)$ & \\
\hline Branching & $2(6.7)$ & $0(0.0)$ & & $1(3.3)$ & $2(2.3)$ & \\
\hline Penetrating \& Branching & $3(10.0)$ & $0(0.0)$ & & $16(53.3)$ & $0(0.0)$ & \\
\hline Penetrating \& Branching \& Shunt & $0(0.0)$ & $0(0.0)$ & & $7(23.3)$ & $0(0.0)$ & \\
\hline Distribution & & & 0.269 & & & 0.220 \\
\hline N/A & $2(6.7)$ & $19(22.1)$ & & $1(3.3)$ & $16(18.6)$ & \\
\hline Peripheral & $12(40.0)$ & $33(38.4)$ & & $4(13.3)$ & $10(11.6)$ & \\
\hline Central & $3(10.0)$ & $6(7.0)$ & & 5 (16.7) & $15(17.4)$ & \\
\hline Both & $13(43.3)$ & $28(32.6)$ & & $20(66.7)$ & $45(52.3)$ & \\
\hline
\end{tabular}

${ }^{\mathrm{a}} \mathrm{P}<0.05$.

Table IV. Comparison of the diagnostic performance of US, US+CDFI and US+SMI.

\begin{tabular}{lcccccr}
\hline Variable & Sensitivity $(\%)$ & Specificity $(\%)$ & Accuracy $(\%)$ & AUC & $95 \%$ CI & P-value \\
\hline US & 73.33 & 66.28 & 68.10 & 0.698 & $0.589-0.807$ & \\
US + CDFI & 80.00 & 72.09 & 74.14 & 0.760 & $0.660-0.860$ & $<0.001$ \\
US + SMI & 86.67 & 83.72 & 84.48 & 0.852 & $0.768-0.936$ & \\
\hline
\end{tabular}

US, ultrasonography; CDFI, color Doppler flow imaging; SMI, superb microvascular imaging; AUC, area under ROC; CI, confidence interval .

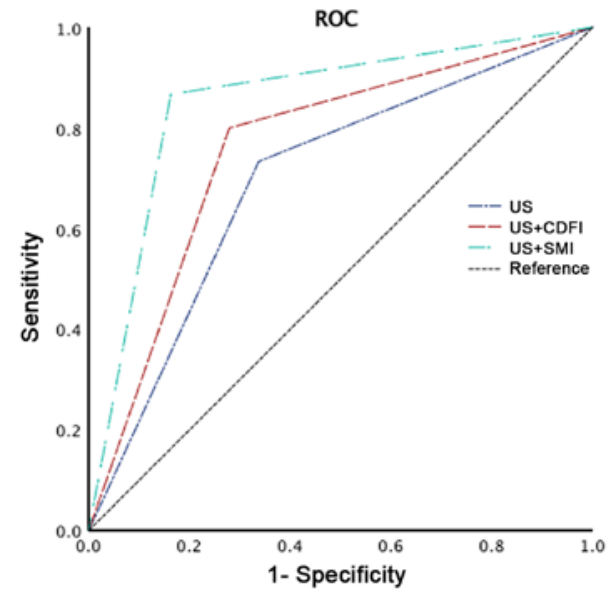

Figure 5. BI-RADS category grading based on US findings, US + CDFI findings, and US+SMI findings. BI-RADS, Breast Imaging Reporting and Data System; US, ultrasonography; CDFI, color Doppler flow imaging; SMI, superb microvascular imaging; ROC, receiver operating curve. and 1 papilloma were observed to be delineated with penetrating and branching vessels, respectively under SMI examination. When integrating with other vascular characteristics, the two benign breast lesions were upgraded from $4 \mathrm{a}$ to $4 \mathrm{c}$ and $4 \mathrm{~b}$, respectively. Furthermore, calcifications in the hyperechoic area may result in the misdiagnosis of true negative cases.

There were certain limitations to the present study. Firstly, the study was only conducted in one center with limited pathological groups. Second, all the examinations were conducted by one radiologist and, consequently, there was no interpretation of inter-observer differences. Third, the inclusion of samples may be biased, as BI-RADS category 3 breast masses were not included in the present study, although none of the examined breast masses were downgraded to category 3 neither by CDFI or SMI. Therefore, further research should include larger samples, from multiple centers and include the full scale of BI-RADS categories. 
In summary, the present study compared SMI with CDFI to evaluate vascular quantity, morphology and distribution for differentiating between malignant and benign BI-RADS 4 category breast lesions. SMI was able to overcome the shortcomings of CDFI in detecting low-velocity blood flow due to motion artifacts. Our findings demonstrated that SMI is superior to CDFI in identifying and characterizing vascular details further. We also reported that, as an adjunct to grayscale US, SMI exhibited notable diagnostic performance in distinguishing between malignant and benign BI-RADS category 4 breast lesions.

\section{Acknowledgements}

Not applicable.

\section{Funding}

The present study was funded by Important Weak Subject Construction Project of Pudong Health and Family Planning Commission of Shanghai (Shanghai, China; grant no. PWzbr 2017-10).

\section{Availability of data and materials}

The datasets used and/or analyzed during the present study are available from the corresponding author on reasonable request.

\section{Authors' contributions}

YCZ designed the study. YZ, SHD and QJ collected and analyzed the data. DMZ, JS and XRS contributed the collection of samples and provided intellectual input. YCZ was a major contributor in writing the manuscript. All authors read and approved the final manuscript.

\section{Ethics approval and consent to participate}

The Ethics Committee of Shanghai Pudong New Area People's Hospital (Shanghai, China) approved the prospective study. All enrolled patients were notified of the examinations and procedure, and written informed consents was provided by all patients.

\section{Patient consent for publication}

Not applicable.

\section{Competing interests}

The authors declare that they have no competing interests.

\section{References}

1. Li T, Mello-Thoms C and Brennan PC: Descriptive epidemiology of breast cancer in China: Incidence, mortality, survival and prevalence. Breast Cancer Res Treat 159: 395-406, 2016.

2. Rezaee A, Buck A, Raderer M, Langsteger W and Beheshti M: Chapter 3-breast cancer BT-PET/CT in cancer: An interdisciplinary approach to individualized imaging. Elsevier, pp43-63, 2018.
3. Folkman J: Angiogenesis in cancer, vascular, rheumatoid and other disease. Nat Med 1: 27-31, 1995.

4. Stratman AN, Yu JA, Mulligan TS, Butler MG, Sause ET and Weinstein BM: Chapter 24-blood vessel formation, editor(s): Sally a. Moody, Principels of developmental genetics (Second edition), Academic Press, pp421-449, 2015.

5. Lee SH, Chung J, Choi HY, Choi SH, Ryu EB, Ko KH, Koo HR, Park JS, Yi A, Youk JH, et al: Evaluation of screening US-detected breast masses by combined use of elastography and color doppler US with B-mode US in women with dense breasts: A multicenter prospective study. Radiology 285: 660-669, 2017

6. Kook SH, Park HW, Lee YR, Lee YU, Pae WK and Park YL: Evaluation of solid breast lesions with power Doppler sonography. J Clin Ultrasound 27: 231-237, 1999.

7. Park AY, Seo BK, Woo OH, Jung KS, Cho KR, Park EK, Cha SH and Cha J: The utility of ultrasound superb microvascular imaging for evaluation of breast tumour vascularity: Comparison with colour and power Doppler imaging regarding diagnostic performance. Clin Radiol 73: 304-311, 2018.

8. Zhu YC, Zhang Y, Deng SH and Jiang Q: A prospective study to compare superb microvascular imaging with grayscale ultrasound and color doppler flow imaging of vascular distribution and morphology in Thyroid nodules. Med Sci Monit 24: 9223-9231, 2018.

9. Dubinsky TJ, Revels J, Wang S, Toia G, Sonneborn R, Hippe DS and Erpelding T: Comparison of Superb Microvascular Imaging with color flow and power doppler imaging of small hepatocellular carcinomas. J Ultrasound Med 37: 2915-2924, 2018.

10. He MN, Lv K, Jiang YX and Jiang TA: Application of superb microvascular imaging in focal liver lesions. World J Gastroenterol 23: 7765-7775, 2017.

11. Mercado CL: BI-RADS update. Radiol Clin North Am 52: 481-487, 2014

12. Levy L, Suissa M, Chiche JF, Teman G and Martin B: BIRADS ultrasonography: Eur J Radiol 61: 202-211, 2007.

13. Strigel RM, Burnside ES, Elezaby M, Fowler AM, Kelcz F, Salkowski LR and DeMartini WB: Utility of BI-RADS assessment category 4 subdivisions for screening breast MRI. AJR Am J Roentgenol 208: 1392-1399, 2017.

14. Raza S, Goldkamp AL, Chikarmane SA and Birdwell RL: US of breast masses categorized as BI-RADS 3, 4, and 5: Pictorial review of factors influencing clinical management. Radiographics 30: 1199-1213, 2010.

15. Adler DD, Carson PL, Rubin JM and Quinn-Reid D: Doppler ultrasound color flow imaging in the study of breast cancer: Preliminary findings. Ultrasound Med Biol 16: 553-559, 1990.

16. Durand MA and Hooley RJ: Implementation of whole-breast screening ultrasonography. Radiol Clin North Am 55: 527-539, 2017.

17. Niell BL, Freer PE, Weinfurtner RJ, Arleo EK and Drukteinis JS: Screening for breast cancer. Radiol Clin North Am 55: 1145-1162, 2017.

18. Thomas L and Szabo: Chapter 11-Doppler modes in diagnostic ultrasound imaging: Inside out (Second edition). Academic Press, pp 431-500, 2014.

19. Lu R, Meng Y, Zhang Y, Zhao W, Wang X, Jin M and Guo R: Superb microvascular imaging (SMI) compared with conventional ultrasound for evaluating thyroid nodules. BMC Med Imaging 17: 65, 2017.

20. Durmaz MS and Sivri M: Comparison of superb micro-vascular imaging (SMI) and conventional Doppler imaging techniques for evaluating testicular blood flow. J Med Ultrason 45: 443-452, 2001.

21. Lee DH, Lee JY and Han JK: Superb microvascular imaging technology for ultrasound examinations: Initial experiences for hepatic tumors. Eur J Radiol 85: 2090-2095, 2016.

22. Ma Y, Li G, Li J and Ren WD: The diagnostic value of superb microvascular imaging (SMI) in detecting blood flow signals of breast lesions: A preliminary study comparing SMI to color doppler flow imaging. Medicine (Baltimore) 94: e1502, 2015.

23. Zhu YC, Zhang Y, Deng SH and Jiang Q: Diagnostic performance of superb microvascular imaging (SMI) combined with shear-Wave elastography in evaluating breast lesions. Med Sci Monit 24: 5935-5942, 2018.

24. Less JR, Skalak TC, Sevick EM and Jain RK: Microvascular architecture in a mammary carcinoma: Branching patterns and vessel dimensions. Cancer Res 51: 265-273, 1991.

25. Williams RG: The vascularity of normal and neoplastic grafts in vivo. Cancer Res 11: 139-144, 1951. 
26. Kopeć $M$ and Abramczyk H: Angiogenesis-a crucial step in breast cancer growth, progression and dissemination by Raman imaging. Spectrochim Acta Part A Mol Biomol Spectrosc 198: 338-345, 2018.

27. Raza S and Baum JK: Solid breast lesions: Evaluation with power Doppler US. Radiology 203: 164-168, 1997.

28. Xiao XY, Chen X, Guan XF, Wu H, Qin W and Luo BM: Superb microvascular imaging in diagnosis of breast lesions: A comparative study with contrast-enhanced ultrasonographic microvascular imaging. Br J Radiol 89: 20160545, 2016.

29. Zhan J, Diao XH, Jin JM, Chen L and Chen Y: Superb microvascular imaging-A new vascular detecting ultrasonographic technique for avascular breast masses: A preliminary study. Eur J Radiol 85: 915-921, 2016.
30. Yen PL, Wu HK, Tseng HS, Kuo SJ, Huang YL, Chen HT and Chen DR: Vascular morphologic information of three-dimensional power Doppler ultrasound is valuable in the classification of breast lesions. Clin Imaging 36: 267-271, 2012.

This work is licensed under a Creative Commons Attribution-NonCommercial-NoDerivatives 4.0 International (CC BY-NC-ND 4.0) License. 